\title{
In-Hospital Outcome of Right Ventricular Involvement in Patients with Acute Inferior Myocardial Infarction
}

\author{
Ahmad Bahie Elden ${ }^{1, ~ *, ~ M a h m o u d ~ A l i ~ M a h m o u d ~ A s h r y ~}{ }^{1}$, Mostafa Abdullah Haridi², \\ Sara Hussein Abdelrazek ${ }^{3}$ \\ ${ }^{1}$ Critical Care Unit, Department of Internal Medicine, Assiut University, Assiut City, Egypt \\ ${ }^{2}$ Endocrinology Unit, Department of Internal Medicine, Assiut University, Assiut City, Egypt \\ ${ }^{3}$ Department of Internal Medicine, Assiut University, Assiut City, Egypt \\ Email address: \\ ahmadbahie@yahoo.com (A. B. Elden),mamashry@yahoo.com (M. A. M. Ashry), mharidi2006@yahoo.com (M. A. Haridi), \\ sarahussein43@yahoo.com (S. H. Abdelrazek) \\ *Corresponding author
}

\section{To cite this article:}

Ahmad Bahie Elden, Mahmoud Ali Mahmoud Ashry, Mostafa Abdullah Haridi, Sara Hussein Abdelrazek. In-Hospital Outcome of Right Ventricular Involvement in Patients with Acute Inferior Myocardial Infarction. American Journal of Internal Medicine. Vol. 5, No. 2, 2017, pp. 22-25. doi: 10.11648/j.ajim.20170502.12

Received: March 12,2017; Accepted: March 20, 2017; Published: April 13, 2017

\begin{abstract}
Aim of the Work: To study the in-hospital outcome of right ventricular involvement (RVI) in patients with acute inferior myocardial infarction (AIMI). Material and Methods: We conducted a short prospective study carried out on 60 patients with AIMI, classified into two groups; patients group: AIMI with RVI; inferior or infroposterior acute myocardial infarction (AMI) with $\geq 0.1 \mathrm{mV}$ V4R elevation, and control group: AIMI without RVI; inferior or infroposterior AMI without $\geq 0.1 \mathrm{mV}$ V4R elevation. We followed up both groups during their hospital stay and compared both groups regarding development of complications and mortality. Results: Patients with RVI in AIMI had more admission hyperglycaemia (268.80 $\pm 81.88 \mathrm{mg} / \mathrm{dl}$ vs. $219.80 \pm 98.40 \mathrm{mg} / \mathrm{dl}$, P value $=0.003)$, less incidence of complete ST elevation resolution $(\mathrm{STR})(60.8 \%$ vs. $80.6 \%$, p value $=0.032$ ), more arrhythmic complications (3rd heart block $[\mathrm{HB}]: 6.7 \%$ vs. $0.0 \%, \mathrm{p}$ value $=0.042$, atrial fibrillation [AF]: $10.0 \%$ vs. $2.0 \%$, p value $=0.036$ ). Conclusions: Patients with RVI in AIMI had worse in-hospital outcome than patients without RVI in the form of more admission hyperglycemia, less incidence of complete STR and more arrhythmic complications.
\end{abstract}

Keywords: Right Ventricular Involvement, Acute Inferior Myocardial Infarction, Right Ventricular Infarction

\section{Introduction}

About $30 \%$ to $50 \%$ of patients with AIMI have RVI [1]. Hemodynamically significant RVI is presented with hypotension, elevated jugular venous pulse (JVP), and occasionally shock, all in the presence of clear lung fields [2] The ST-segment elevation in the right precordial leads (V4R) of the Electrocardiograph (ECG) of $\geq 0.1 \mathrm{mV}$ is a documented sign for diagnosis of RVI [3]. The outcome of RV involvement in AIMI patients was not extensively studied and need more thorough follow up. The aim of this study was to study the outcome of RV involvement in AIMI patients.

\section{Patients and Methods}

\subsection{Methodology}

We conducted an observational study with control group, carried out on 60 patients with AMI admitted in internal medicine department CCU at Assiut University Hospital, between January 2016 and May 2016. Written consents were obtained from most of the participants; illiterate participants gave their consent by finger prints. We included patients presented by inferior or infroposterior AMI within $12 \mathrm{~h}$ from symptom onset, treated by thrombolytic agents, with at least $50 \%$ resolution of ST segment elevation and infarct zone comprised at least three hypokinetic LV inferior or 
infroposterior walls segments by echocardiography. The diagnosis of inferior or infroposterior AMI was based on prolonged chest pain lasting $\geq 30 \mathrm{~min}$, ST segment elevation $\geq$ $1 \mathrm{~mm}$ in at least two electrocardiographic (ECG) inferior leads with or without ST segment depression $\geq 1 \mathrm{~mm}$ of $\mathrm{V}_{1}$ to $\mathrm{V}_{3}$, and a more than threefold increase in serum creatine kinase (CK) level [4]. RV involvement defined as ST-segment elevation of $\geq 0.1 \mathrm{mV}$ in any number of right precordial leads including $V_{4} R$. We then classified reperfused patients into two groups; completely reperfused with resolution of ST elevation by $>70 \%$ [5] and partially reperfused with ST resolution by $>$ $50-70 \%$. We excluded patients with inadequate echocardiographic image quality, significant valvular disease, prior Q-wave MI, presence of LBBB. All patients received treatment of AMI according to 2007 Focused Update of the ACC/AHA 2004 Guidelines for the Management of Patients with ST-Elevation Myocardial Infarction [6].

All echocardiograms were performed with an HDI 5000 instrument (Philips Medical Systems, Bothell, Washington, USA) equipped with a broad band harmonic transducer. A standard echocardiography was used within the $3 \pm 2$ days of admission based on apical four and two-chamber views; 2D echocardiograms of the LV short axis were recorded at three levels: mitral valve, mid-papillary muscle level, and apex. All echocardiograms were analyzed at Assiut University Internal Medicine Echo Laboratory. LV dimensions and ejection fraction (EF) was calculated using M Mode. The mean value of three measurements of the technically best cardiac cycles was taken from each examination. To evaluate regional systolic function, the left ventricle was divided according to a 16-segment model as recommended by the American Society of Echocardiography 2002. For each segment, wall motion was scored from 1 (normal) to 4 (dyskinetic) and a global segmental wall motion score (SWMS) was calculated as the average over 16 segments [7].

\subsection{Classification of Patients}

We classified our recruit into two groups; patients group: AIMI with RVI; inferior or infroposterior acute myocardial infarction (AMI) with $\geq 0.1 \mathrm{mV}$ V4R elevation, and control group: AIMI without RVI; inferior or infroposterior AMI without $\geq 0.1 \mathrm{mV}$ V4R elevation.

\subsection{Statistical Analysis}

The statistical analysis was performed using SPSS (version 16.0, SPSS Inc., Chicago, IL, USA). The KolmogorovSmirnov test was used to test normally. The continuous variables were presented as the means $\pm \mathrm{SD}$ and categorical variables were reported as percentages. Student T-test was used to compare between means of 2 continuous variables in case of parametric data and Mann-Whitney test for non-parametric data, and chi-square test was used to compare percentages and ratios. Pearson correlation was done to measure correlation among quantitative variables. A p-value $<$ 0.05 was considered statistically significant.

\section{Results}

\subsection{Basal Characteristics}

The study carried out on 60 patients. 30 patients had AIMI with RVI and 30 patients had AIMI without RVI. Patients' characteristics of both groups are shown in Table 1. The study showed insignificant differences between groups as regard age, gender, BMI and risk factors. There were significant differences between groups in admission systolic and diastolic BP. Patients without RVI had significantly higher HR than patients with RVI.

\subsection{Laboratory Data}

There were insignificant differences in peak CK, troponin, LDL, HDL, serum creatinine and BUN. Patients with RVI had significantly higher admission BG level than patients without RVI as in Table 1.

\subsection{ECG Data}

There is insignificant difference between groups in ECG infarction size estimated by number of involved leads and summation of ST elevations in LV leads. There were significantly higher incidences in atrial fibrillation and third degree heart block in patients with RVI and there was significantly higher rate of $>70 \%$ resolution in STE in patients without RVI as in Table 2.

\subsection{Echocardiographic Data}

There were insignificant differences between groups in EF and SWMSI, PASP, incidence of mitral regurgitation (MR) and tricuspid regurgitation (TR) as in Table 2.

Table 1. Demographic, Selected Clinical and Laboratory Data.

\begin{tabular}{llll}
\hline & $\begin{array}{l}\text { Patient with RVI } \\
(\mathbf{n = 3 0})\end{array}$ & $\begin{array}{l}\text { Patients without } \\
\text { RVI }(\mathbf{n = 3 0})\end{array}$ & P-value \\
\hline Age: & $55.83 \pm 9.44$ & $54.87 \pm 13.35$ & 0.583 \\
Male & $23(76.7 \%)$ & $27(90.0 \%)$ & 0.166 \\
Duration of admission: & $4.73 \pm 1.39$ & $4.53 \pm 1.14$ & 0.095 \\
Hx. DM & 63.3 & 40.0 & 0.071 \\
Hx. HTN & 26.7 & 30.0 & 0.774 \\
C. Smoking & 76.7 & 83.3 & 0.519 \\
BMI & $30 \pm 2.2$ & $29 \pm 3.1$ & 0.167 \\
Systolic BP: & $90.00 \pm 28.13$ & $129.33 \pm 20.16$ & 0.002 \\
Diastolic BP: & $69.00 \pm 18.45$ & $81.67 \pm 11.17$ & 0.031 \\
Heart rate: & $71.33 \pm 16.91$ & $82.53 \pm 12.29$ & 0.006 \\
Total CK: & $1129.93 \pm 779.16$ & $1642.60 \pm 1157.86$ & 0.095 \\
Troponin & $0.93 \pm 0.94$ & $4.25 \pm 8.14$ & 0.264 \\
Random blood sugar: & $268.80 \pm 81.88$ & $219.80 \pm 98.40$ & 0.003 \\
LDL: & $130.93 \pm 37.68$ & $130.12 \pm 43.24$ & 0.667 \\
HDL: & $30.90 \pm 8.24$ & $32.87 \pm 8.52$ & 0.372 \\
BUN: & $5.99 \pm 3.25$ & $5.58 \pm 3.19$ & 0.673 \\
S. creatinine: & $97.90 \pm 31.93$ & $98.76 \pm 43.25$ & 0.625 \\
\hline
\end{tabular}

N: number, h: hour, HTN: hypertension, DM: diabetes mellitus, BMI: body mass index, HR: heart rate, BP: blood pressure; sum ST, summation of ST segment elevation, S CR: serum creatinine. 
Table 2. ECG, Echo and mortality data.

\begin{tabular}{llll}
\hline & $\begin{array}{l}\text { Patient with } \\
\text { RVI (n= 30) }\end{array}$ & $\begin{array}{l}\text { Patients without } \\
\text { RVI (n= 30) }\end{array}$ & P-value \\
\hline ECG: & & & \\
Sum ST & $13.5 \pm 4.5$ & $12.5 \pm 5.5$ & 0.063 \\
Number of LV leads & $4 \pm 2$ & $4 \pm 3$ & 0.987 \\
$>70 \%$ resolution (\%) & $60.8 \%$ & $80.6 \%$ & 0.032 \\
Arrhythmia: & & & \\
$3^{\text {rd }}$ HB & 6.7 & 0.0 & 0.042 \\
$1^{\text {st }}$ HB & 11.0 & 13.3 & 0.121 \\
Atrial Fib & 10.0 & 2.0 & 0.036 \\
VT & 6.7 & 6.7 & -- \\
ECHO & & & 0.27 \\
EF: & $55.63 \pm 10.22$ & $55.03 \pm 10.41$ & 0.051 \\
SWMSI: & $1.22 \pm 0.15$ & $1.21 \pm 0.16$ & 0.310 \\
PASP: & $38.73 \pm 7.63$ & $37.37 \pm 5.30$ & 0.573 \\
MR No. $(\%)$ & $10(33.3 \%)$ & $8(26.7 \%)$ & 0.000 \\
TR No. $(\%)$ & $22(73.3 \%)$ & $13(76.7 \%)$ & 0.472 \\
Death: & $2(6.7 \%)$ & $0(0.0 \%)$ & \\
\hline
\end{tabular}

Sum ST; summation of ST segment elevation, LV; left ventricle, RV; right ventricle, STR; ST resolution, HB; heart block, atrial fib; atrial fibrillation, EF; ejection fraction, WMSI; wall motion score index, PASP; pulmonary artery systolic pressure, MR; mitral regurgitation, TR; tricuspid regurgitation.

\section{Discussion}

Our study groups were homogenous as regard age, gender, and risk factors, LDL, HDL, serum creatinine, BUN, LV infarction size (ECG, enzymes and echocardiography driven) and LVEF, which reduced confounding factors. In a meta-analysis of the published literatures, it has been reported that stress hyperglycemia $(\mathrm{SH})$ was associated with an increased risk of mortality in AMI irrespective of diabetic status [8]. It has been proposed that in AMI patients, decreased levels of blood insulin make the heart has to depend on free fatty acids for its metabolism. The accumulation of excessive fatty acids results in the reduction of myocardial contractility and increases the risk of pump failure and arrhythmias [9]. Acute illness, raise levels of counter regulatory hormones resulting in increased hepatic glucose production, impaired peripheral glucose utilization [10]. In our study, patients with RVI had significantly higher admission BG level than patients without RVI which can carry a bad prognosis.

Monitoring of ST-segment resolution in the surface ECG is the simplest way of determining adequacy of myocardial reperfusion. The most common method of STR classification is the following: incomplete; ST-segment resolution $<30 \%$, partial; ST-segment resolution $30-70 \%$ and complete; ST-segment resolution $\geq 70 \%$ [11]. Angeja et al. showed that angiographic assessment of reperfusion provides information on the mechanical patency of epicardial arteries, while the surface electrocardiography reflects the functional status of myocardium perfusion [12]. There was significantly lower rate of $>70 \%$ STR in patients with RVI. Partial reperfusion with $>50-<70 \%$ resolution in STE is associated with no reflow phenomenon. No-reflow has a strong negative impact upon clinical outcome [13]. No-reflow is associated with higher prevalence of early post infarct complications, adverse
LV remodeling, repeat hospital stays for heart failure and mortality [14]. No-reflow is caused by distal atherothrombotic embolization, ischemic injury, reperfusion injury and heightened susceptibility of coronary microcirculation to injury [15].

RVMIs are more often complicated by all types of arrhythmias compared with 'simple' inferior AMIs [16]. Barrillon et al [17] were the first to recognize the significantly higher risk of conduction disorders in patients with RVI. Complete atrioventricular (AV) or sinoatrial blocks occurred in one half of cases in which STE in V4R were present. Zehender et al [18] demonstrated a higher incidence of sustained ventricular tachycardia and ventricular fibrillation in patients with ECG signs of RVI and significantly higher incidences of complete AV block and severe bradycardias with pacing requirements have been reported in cases of RVI [18]. In our study, there were significantly higher incidences in atrial fibrillation and third degree heart block in patients with RVI. Patients with RVI had insignificantly higher mortality than patients with without. However, the small sample size and the short duration of follow up could explain the statistical insignificance.

Higher ST segment elevations in V4R have been found to be independent predictive factors for more significant $R V$ dysfunction and higher mortality rates [18]. In our study, despite there was ECG characters of RVI in patient group, there were insignificant differences between groups regarding; PASP, incidence of MR and TR.

\section{Conclusion}

We concluded that patients with RVI in AIMI had worse in-hospital outcome than patients without RVI in the form of more admission hyperglycemia, less incidence of complete STR and more arrhythmic complications. We recommend a more frequent blood glucose monitoring, extensive glycaemic control, early revascularization and more extensive arrhythmic control to limit complications in RVI patients.

\section{Study Limitations}

Financial factors interfering with performing a diagnostic coronary angiography in the admission days, so we depended on ST segment resolution only; this was used to reflect myocardial perfusion rather than epicardial perfusion.

\section{References}

[1] Stanley George, Mansi Patel and Ashok Thakkar (2014): Clinical Profile and In-Hospital Outcome of Patients with Right Ventricular Myocardial Infarction. International Journal of Clinical Medicine; 5, 459 - 63.

[2] Haji, S. A. and Movahed, A. (2000): Right Ventricular Infarction-Diagnosis and Treatment. Clinical Cardiology; 23, 473- 482. 
[3] Mehta, S. R., Eikelboom, J. W., Natarajan, M. K., Diaz, R., Yi, C., Gibbons, R. J. and Yusuf, S. (2001): Impact of Right Ventricular Involvement on Mortality and Morbidity in Patients with Inferior Myocardial Infarction. Journal of the American College of Cardiology; 37, 37-43.

[4] Elliott M. A, Daniel T. Anbe and Frederick G. Kushner et al. (2004): ACC/AHA Guidelines for the Management of Patients with ST-Elevation Myocardial Infarction. A Report of the American College of Cardiology/American Heart Association Task Force on Practice Guidelines (Committee to Revise the 1999 Guidelines for the Management of Patients With Acute Myocardial Infarction) Developed in Collaboration With the Canadian Cardiovascular Society. American College of Cardiology Web Site (www.cardiosource.org/acc).

[5] Schröder K, Wegscheider K and Zeymer U et al. (2001): Extent of ST-segment deviation in the single ECG lead of maximum deviation present 90 or 180 minutes after start of thrombolytic therapy best predicts outcome in acute myocardial infarction. Zeitschriftfür Kardiologie; 90: 557-67.

[6] Elliott M. A, Hand M and Armstrong P. W et al. (2008): 2007 Focused update of the ACC/AHA 2004 guidelines for the management of patients with ST-elevation myocardial infarction: a report of the American College of Cardiology/ American Heart Association task force on practice guidelines. Circulation; 117 (2): 296-329.

[7] Manuel D. Cerqueira, Neil J. Weissman and Vasken Dilsizian et al. (2002): Standardized Myocardial Segmentation and Nomenclature for Tomographic Imaging of the Heart. American Heart Association, Writing Group on Myocardial Segmentation and Registration for Cardiac Imaging. Circulation; 105: 539-42.

[8] Capes SE, Hunt D and Malmberg K et al. (2000): Stress hyperglycemia and increased risk of death after myocardial infarction in patients with and without diabetes: a systematic overview. Lancet; 356: 773-8.

[9] Oliver M. F and Opie L. H. (1994): Effects of glucose and fatty acids on myocardial ischemia and arrhythmias. The Lancet;
343 (8890): 155-58.

[10] Umpierrez GE and A. E. K. (2004): ICU care for patients with diabetes. Current Opinion in Endocrinology and Diabetes; 11: 75-81.

[11] Bolognese L, Carrabba N and Parodi G et al. (2004): Impact of microvascular dysfunction on left ventricular remodeling and long-term clinical outcome after primary coronary angioplasty for acute myocardial infarction. Circulation; 109: 1121-6.

[12] Angeja BG, Gunda M and Murphy SA et al. (2002): TAIMI myocardial perfusion grade and ST segment resolution: association with infarct size as assessed by single photon emission computed tomography imaging. Circulation; 105: $282-5$.

[13] Brosh D, Assali AR and MagerA et al. (2007): Effect of no-reflow during primary percutaneous coronary intervention for acute myocardial infarction on six-month mortality. American Journal of Cardiology; 99: 442-5.

[14] Bolognese L, Carrabba N and Parodi G et al. (2004): Impact of microvascular dysfunction on left ventricular remodeling and long-term clinical outcome after primary coronary angioplasty for acute myocardial infarction. Circulation; 109: 1121-6.

[15] Niccoli G, Burzotta F and Galiuto L et al. (2009): Myocardial no-reflow in humans. Journal of the American College of Cardiology; 54: 281-92.

[16] Mehta SR, Eikelboom JW and Natarajan MK et al. (2001): Impact of right ventricular involvement on mortality and morbidity in patients with inferior myocardial infarction. Journal of the American College of Cardiology; 37: 37-43.

[17] Barrillon A, Chaignon M, Guize L and Gerbaux A (1975): Premonitory sign of heart block in acute posterior myocardial infarction. British Heart Journal; 37: 2-8.

[18] Zehender M, Kasper W and Kauder E et al. (1993): Right ventricular infarction as an independent predictor of prognosis after acute inferior myocardial infarction. New England Journal of Medicine; 328: 981-8. 